\title{
Tabagismo entre estudantes de profissões de saúde: prevalência, conhecimento, atitudes e opiniões ${ }^{\mathrm{a}}$
}

\author{
Smoking among health professional students: prevalence, knowledge, attitudes and opinions
}

\author{
Anderson Cardoso da Silva ${ }^{\dagger *}$, Eduardo Ribeiro Teixeira ${ }^{\dagger}$, Sebastião Jorge da Cunha Gonçalves", \\ Maria Cristina Almeida de Souza ${ }^{\S}$
}

Como citar esse artigo. Da Silva AC, Texeira ER, Gonçalvez SJC, de Souza MCA. Tabagismo entre estudantes de profissões de saúde: prevalência, conhecimento, atitudes e opiniões. Revista de Saúde. 2017 Jan./Jun.; 08 (1): 23-27.

\begin{abstract}
Resumo
O tabagismo é a principal causa evitável de mortalidade no mundo. Cabe ao pessoal de saúde empenhar-se para mudar isso, mas sua atuação depende de serem ou não tabagistas, e do treinamento recebido. O objetivo deste trabalho foi verificar se a atitude dos estudantes de profissões da saúde é modificada pelo fato de terem hábito de fumar, estimar a prevalência de tabagismo e avaliar opiniões. Trata-se de um estudo transversal, com inquérito aplicado a uma amostra aleatória simples de acadêmicos de medicina, enfermagem, odontologia e farmácia, segundo a metodologia da Pesquisa Mundial sobre Tabagismo em Estudantes de Profissões de Saúde. A análise estatística considerou um valor de $\mathrm{p}<0,05$ como índice de significância. O questionário foi respondido por 114 estudantes; $65,8 \%$ eram do gênero feminino e $72,8 \%$ tinham menos de 24 anos. A prevalência de tabagismo foi de 19,3\%, estando acima da média nacional, e foi maior no gênero masculino. Quanto às opiniões, destaca-se que para 82,6\% dos não fumantes, os profissionais da saúde (PS) servem de modelo de comportamento, contra 59,1\% dos fumantes $(\mathrm{p}=0,02)$; para $97,8 \%$ dos não fumantes, os PS devem rotineiramente aconselhar pacientes a parar de fumar, contra $81,8 \%$ dos fumantes $(\mathrm{p} \leq 0,01)$. A maioria respondeu que PS devem receber treinamento sobre técnicas de cessação, mas 75,4\% afirmam não terem sido treinados. Considerando a alta prevalência de tabagismo e seu impacto sobre a atuação dos futuros profissionais da saúde, conclui-se que deve ser destinada maior atenção ao treinamento dado sobre abandono do uso de tabaco.
\end{abstract}

Palavras-chave: Abandono do uso de tabaco; Hábito de fumar; Pessoal de saúde; Tabaco.

\begin{abstract}
Smoking is the leading preventable cause of mortality in most countries. It is up to health personnel to commit to changing this situation, but their practice depends on them being smokers themselves and receiving training. The objective of this study was to determine whether the attitude of students from health courses is modified by the fact that they have smoking habits, to estimate the prevalence of smoking and evaluate opinions on the subject of smoking. This is a crosssectional study with a survey applied to a simple random sample of students of medical schools, nursing, dentistry and pharmaceutics, based on the standard questionnaire of the Global Health Professional Students Survey. The analysis considered a p-value $<0.05$ as statistically significant. The questionnaire was completed by 114 students, $65.8 \%$ female and $72.8 \%$ under 24 years of age. The overall prevalence of smoking was $19.3 \%$, above the national average, and higher in males. Concerning the students' opinions, $82.6 \%$ of non-smokers consider health professionals (HP) to be role models, as opposed to $59.1 \%$ of smokers $(\mathrm{p}=0.02) ; 97.8 \%$ of non-smokers believe that HP must routinely advise their patients to stop smoking, as opposed to $81.8 \%$ of smokers $(\mathrm{p} \leq 0.01)$. Although most students have answered that HP should receive training on cessation techniques, $75.4 \%$ said they had not received any formal training. Considering the high prevalence of smoking and its impact on the practice of future health professionals, it is necessary to give more attention to the training given on tobacco use cessation.
\end{abstract}

Keywords: Tobacco Use Cessation; Smoking; Health Personnel; Tobacco.

\section{Introdução}

Apesar do avanço das iniciativas de combate ao seu consumo, o tabaco continua sendo a principal causa global evitável de morte. A Organização Mundial de Saúde (OMS) estima que por ano mais de 6 milhões de pessoas morram e que ocorram prejuízos econômicos superiores a meio trilhão de dólares em decorrência do consumo de tabaco em todo o mundo ${ }^{1}$.

Nos próximos 50 anos, o tabagismo deve causar cerca de 450 milhões de mortes em todo o mundo. Reduzindo-se as atuais taxas de prevalência pela metade, cerca de 20 a 30 milhões de mortes prematuras poderiam ser evitadas só no primeiro quarto de século,

Afiliação dos autores: † Universidade Severino Sombra, Pró-Reitoria de Ciências Médicas, Discente do Curso de Medicina, Vassouras, RJ, Brasil

¥ Universidade Severino Sombra, Pró-Reitoria de Ciências Médicas, Docente do Curso de Medicina, Vassouras, RJ, Brasil

$\S$ Universidade Severino Sombra, Pró-Reitoria de Ciências Médicas, Docente do Curso de Medicina e do Mestrado Profissionalizante em Ciências Aplicadas em Saúde, Vassouras,

RJ, Brasil 
e 150 milhões no segundo. Prevenindo-se que jovens iniciem o consumo de tabaco será possível reduzir o número de mortes (por causas relacionadas) apenas após $2050^{2}$. Dessa forma, a cessação do tabagismo é a única maneira de diminuir a mortalidade por causas relacionadas em médio prazo. $\mathrm{E}$ os profissionais da saúde têm um papel fundamental nesse objetivo.

Evidências demonstram que intervenções feitas com pacientes tabagistas, ainda que curtas (menos de 3 minutos) podem fazer diferença, existindo inclusive, uma relação direta entre a intensidade da intervenção e os resultados na cessação. Mesmo aqueles que no momento da consulta não estejam inclinados a parar de fumar são, de alguma forma, influenciados ${ }^{3}$.

A prevalência do uso do tabaco entre os profissionais da área da saúde é preocupante, pois esse é um dos fatores que pode prejudicar sua atuação no controle adequado do tabagismo. Além do fato de que esses profissionais, sobretudo médicos, dão um exemplo de vida para os pacientes, são eles os que deveriam combater com maior capacidade e responsabilidade essa doença ${ }^{4}$.

A partir de observações nos diversos cenários em que os acadêmicos do curso de medicina da Universidade Severino Sombra (USS) interagem com pacientes, levantou-se a hipótese de que a conduta desses futuros profissionais da saúde frente ao paciente que utiliza tabaco, depende não apenas do treinamento formal que recebem, mas também se eles próprios são ou não tabagistas. Estende-se a hipótese aos demais cursos da área da saúde.

O objetivo deste trabalho foi verificar se a atitude dos estudantes de cursos da área da saúde da USS frente ao paciente que fuma é ou não modificada pelo fato de o aluno ser ou não tabagista, estimar a prevalência de consumo de cigarros e outros produtos derivados do tabaco, determinar o perfil demográfico e tabágico dos participantes e avaliar como o currículo escolar contempla o tema, inclusive o treinamento formal dos acadêmicos para orientar os pacientes sobre os riscos do tabagismo, bem como a aquisição de técnicas para auxiliar na cessação.

\section{Materiais e métodos}

Trata-se de um estudo transversal, cuja amostra foi composta por estudantes dos cursos de medicina, enfermagem, odontologia e farmácia da USS, situada no município de Vassouras, estado do Rio de Janeiro. Realizado entre os meses de junho e julho de 2016, utilizou a metodologia padronizada do Global Health Professional Students Survey (GHPSS), que se baseia em acadêmicos do terceiro ano, haja visto que na maioria dos países esses estudantes estão em níveis avançados do seu treinamento, sem necessariamente já estarem incluídos nos cenários clínicos. ${ }^{5}$

A GHPSS, que integra o Sistema Mundial de Vigilância de Tabagismo (Global Tobacco Surveillance System - GTSS), foi desenvolvida em conjunto pela OMS, o Centro de Controle de Doenças dos Estados Unidos e a Associação de Saúde Pública Canadense. O seu questionário padrão é composto por 42 questões divididas em seis áreas: questões de 1 a 9. Prevalência de uso de tabaco; questões de 10 a 13, Exposição ambiental à fumaça de tabaco; questões de 14 a 24, Atitudes frente ao tabagismo; questões de 25 a 32, Comportamento e cessação; questões de 33 a 39, Currículo e treinamento, e; questões de 40 a 42, Dados demográficos. O questionário fornece às instituições e entidades uma maneira de mensurar o consumo de tabaco entre os estudantes de cursos da área da saúde, o desejo de cessação entre os que fumam, a maneira como vêm sendo treinados em técnicas de aconselhamento para cessação e o desejo de utilizarem esse treinamento para reduzir o uso de tabaco entre seus pacientes. Dessa forma, essa metodologia proporciona o subsídio necessário à elaboração e implementação de medidas que melhor preparem os futuros profissionais da saúde a desempenharem seu papel crucial na luta pela redução do tabagismo ${ }^{5}$.

A quantidade total de estudantes da USS matriculados no quinto e sexto períodos (terceiro ano) foi obtida junto à coordenação de cada curso. No caso de cursos que não tinham turmas de terceiro ano, foram incluídas as que estivessem o mais próximo possível. A partir desses dados, foi calculado o tamanho necessário da amostra para possibilitar intervalo de confiança de $95 \%$, tolerandose um erro amostral de 5\%. Os estudantes foram então escolhidos de modo aleatório para participar da pesquisa. O questionário GHPSS (traduzido para o português, validado e adaptado $)^{6}$ foi respondido por aqueles que concordaram com os objetivos da pesquisa e assinaram o Termo de Consentimento Livre e Esclarecido (TCLE). Os critérios de inclusão foram: o participante ser aluno do curso da área de saúde da USS e consentir em assinar o TCLE.

A pesquisa foi feita nas salas de aula durante as atividades acadêmiccas regulares em datas e horários previamente autorizados pelos professores e coordenadores de cada curso.

Os objetivos e metodologia da pesquisa foram explicados aos estudantes e então lhes foi dado tempo para responderem o questionário auto-administrado. Os participantes marcaram suas respostas em folhas de resposta de múltipla escolha (tipo bolha) criadas com o programa FormReturn 1.6. As folhas eram individualizadas, mas sem identificação dos participantes. As respostas foram extraídas por leitura óptica pelo programa FormReturn 1.6, exportadas 
Tabela 1. Variáveis demográficas e sua associação com o tabagismo numa análise bivariada.

\begin{tabular}{|c|c|c|c|c|c|c|c|}
\hline \multirow{2}{*}{ Variáveis demográficas } & \multicolumn{2}{|c|}{ Total } & \multicolumn{2}{|c|}{ Nãofumantes } & \multicolumn{2}{|c|}{ Fumantes } & \multirow[t]{2}{*}{ Valor de $\mathrm{p}$} \\
\hline & $\mathrm{n}$ & $\%$ & $\mathrm{n}$ & $\%$ & $\mathrm{n}$ & $\%$ & \\
\hline \multicolumn{8}{|l|}{ Idade } \\
\hline$<24$ anos & 83 & 72,81 & 68 & 81,93 & 15 & 18,07 & \multirow{2}{*}{$\mathrm{p} \geq 0,05$} \\
\hline$\geq 25$ anos & 31 & 27,19 & 24 & 77,42 & 7 & 22,58 & \\
\hline \multicolumn{8}{|l|}{ Gênero } \\
\hline Feminino & 75 & 65,79 & 65 & 86,67 & 10 & 13,33 & \multirow{2}{*}{0,02} \\
\hline Masculino & 39 & 34,21 & 27 & 69,23 & 12 & 30,77 & \\
\hline \multicolumn{8}{|l|}{ Ano do curso } \\
\hline $2^{\circ}$ ano & 14 & 12,28 & 12 & 85,71 & 2 & 14,29 & \multirow{4}{*}{$\mathrm{p} \geq 0,05$} \\
\hline $3^{\circ}$ ano & 47 & 41,23 & 37 & 78,72 & 10 & 21,28 & \\
\hline $4^{\mathrm{o}}$ ano & 39 & 34,21 & 31 & 79,49 & 8 & 20,51 & \\
\hline $5^{\circ}$ ano & 14 & 12,28 & 12 & 85,71 & 2 & 14,29 & \\
\hline \multicolumn{8}{|l|}{ Curso } \\
\hline Medicina & 42 & 36,84 & 30 & 71,43 & 12 & 28,57 & \multirow{4}{*}{$\mathrm{p} \geq 0,05$} \\
\hline Enfermagem & 24 & 21,05 & 21 & 87,50 & 3 & 12,50 & \\
\hline Odontologia & 34 & 29,82 & 29 & 85,29 & 5 & 14,71 & \\
\hline Farmácia & 14 & 12,28 & 12 & 85,71 & 2 & 14,29 & \\
\hline
\end{tabular}

Fonte: Dados obtidos pelos autores.

Nota: Valor de $\mathbf{p}<\mathbf{0 , 0 5}$ utilizado como índice de significância estatística.

Tabela 2. Atitudes e opiniões de estudantes de profissões de saúde frente o tabagismo.

\begin{tabular}{|c|c|c|c|c|c|c|c|}
\hline \multirow{2}{*}{$\begin{array}{l}\text { Questões respondidas de modo } \\
\text { afirmativo pelos participantes }\end{array}$} & \multicolumn{2}{|c|}{ Total } & \multicolumn{2}{|c|}{ Não fumantes } & \multicolumn{2}{|c|}{ Fumantes } & \multirow[t]{2}{*}{ p-valor } \\
\hline & $\mathbf{n}$ & $\%$ & $\mathbf{n}$ & $\%$ & $\mathbf{n}$ & $\%$ & \\
\hline $\begin{array}{l}\text { PS deveriam receber treinamento específico } \\
\text { sobre técnicas de cessação? }\end{array}$ & 106 & 92,98 & 89 & 96,74 & 17 & 77,27 & $\mathrm{p} \leq 0,01$ \\
\hline $\begin{array}{l}\text { PS servem como modelos de comportamento } \\
\text { para seus pacientes e para o público em } \\
\text { geral? }\end{array}$ & 89 & 78,07 & 76 & 82,61 & 13 & 59,09 & 0,017 \\
\hline $\begin{array}{l}\text { PS deveriam rotineiramente aconselhar seus } \\
\text { pacientes fumantes a pararem de fumar? }\end{array}$ & 108 & 94,74 & 90 & 97,83 & 18 & 81,82 & $\mathrm{p} \leq 0,01$ \\
\hline $\begin{array}{l}\text { PS deveriam rotineiramente aconselhar seus } \\
\text { pacientes que usam produtos que contêm } \\
\text { tabaco a pararem de usar esses produtos? }\end{array}$ & 110 & 96,49 & 89 & 96,74 & 21 & 95,45 & $\mathrm{p} \geq 0,05$ \\
\hline $\begin{array}{l}\text { PS têm o papel de fornecer conselhos e } \\
\text { informações sobre como parar de fumar? }\end{array}$ & 104 & 91,23 & 83 & 90,22 & 21 & 95,45 & $\mathrm{p} \geq 0,05$ \\
\hline $\begin{array}{l}\text { As chances de um paciente parar de fumar } \\
\text { são maiores se um PS o aconselhar a parar? }\end{array}$ & 88 & 77,19 & 74 & 80,43 & 14 & 63,64 & $\mathrm{p} \geq 0,05$ \\
\hline $\begin{array}{l}\text { PS que fumam são menos propensos a } \\
\text { aconselhar seus pacientes a pararem de } \\
\text { fumar? }\end{array}$ & 70 & 61,40 & 62 & 67,39 & 8 & 36,36 & $\mathrm{p} \leq 0,01$ \\
\hline $\begin{array}{l}\text { PS que usam produtos que contêm tabaco são } \\
\text { menos propensos a aconselhar seus pacientes } \\
\text { a pararem de fumar? }\end{array}$ & 68 & 59,65 & 59 & 64,13 & 9 & 40,91 & 0,046 \\
\hline
\end{tabular}

Fonte: Dados obtidos pelos pesquisadores.

Notas: $P S$ = profissionais de saúde;

Valores em porcentagem relativos a: total, 114; não fumantes, 92; fumantes, 22. 
em tabela para conferência no programa Microsoft Excel 2013 e então transportadas para o programa IBM SPSS Statistics 19.0 para análise estatística. Frequência de distribuição em porcentagem foi índice utilizada para descrever características demográficas e outras variáveis. O teste do qui-quadrado foi aplicado para estimar a presença de associação entre variáveis de interesse, utilizando-se um valor de $\mathrm{p}<$ 0,05 como de significância estatística.

\section{Resultados}

O questionário foi respondido por 114 estudantes. Destes, $65,79 \%(\mathrm{n}=75)$ eram do gênero feminino e $72,81 \%(\mathrm{n}=83)$ tinham menos de 24 anos de idade. A prevalência geral de tabagismo foi de $19,29 \%$, sendo maior no gênero masculino (30,77\% versus $13,33 \%$; $\mathrm{p}=0,02)$. Não houve significância estatística para as demais variáveis pesquisadas (Tabela 1 ).

A Tabela 2 apresenta as questões respondidas pelos participantes para avaliar sua atitude e opiniões em relação ao tabagismo. Comparando-se as respostas dos fumantes com a dos não fumantes, se destaca a diferença estatisticamente significativa para as seguintes questões: $96,74 \%$ dos participantes não fumantes responderam que profissionais da saúde (PS) devem receber treinamento sobre técnicas de cessação, e entre os fumantes, $77,27 \%$ são desta opinião ( $\mathrm{p} \leq 0,01) ; 82,61 \%$ dos não fumantes acham que os PS servem de modelo de comportamento para pacientes e sociedade, contra 59,09\% dos fumantes ( $\mathrm{p}$ $=0,017) ; 97,83 \%$ dos não fumantes acreditam que os PS devem rotineiramente aconselhar seus pacientes que têm hábito de fumar a interromper o uso, contra $81,82 \%$ dos fumantes $(p \leq 0,01)$. Para $67,39 \%$ dos participantes não fumantes, PS que fumam são menos propensos a aconselhar seus pacientes a pararem de fumar, contra $36,36 \%$ dos fumantes $(p \leq 0,01)$. Não houve significância estatística na análise bivariada das demais questões dessa seção.

Apesar da maioria dos acadêmicos $(92,98 \%)$ ter respondido que PS devem receber treinamento sobre técnicas de cessação, $75,44 \%$ afirmam não terem recebido nenhum tipo de treinamento formal sobre abordagens a serem usadas com pacientes; $57,89 \%$ responderam que em nenhuma aula durante o seu curso discutiram as razões pelas quais as pessoas fumam; 47,37\% afirmam não terem aprendido sobre a importância de se fornecer materiais educativos para apoiar a cessação; e 40,35\% afirmam não terem ouvido falar sobre o uso de antidepressivos (como bupropiona) no tratamento para parar de fumar.

\section{Discussão}

Em dados divulgados pelo Ministério da Saúde e pelo Instituto Nacional do Câncer em 2011 o percentual geral de universitários brasileiros que fumava cigarros variou entre 10,50\% (João Pessoa - PB, 2006) e 20,40\% (Juiz de Fora - MG, 2007)7,8. A prevalência geral de tabagismo entre universitários da USS foi de $19,29 \%$, o que a coloca acima da média $(15,02 \%)$ e como a segunda instituição com maior percentual geral de tabagismo entre as pesquisadas. Esta alta prevalência demonstra a importância e a necessidade de se investir em cessação do tabagismo ainda durante os cursos de graduação.

A diferença na prevalência de tabagismo entre gêneros (com percentual maior no masculino) encontrada na USS segue a mesma tendência identificada entre todas as instituições pesquisadas, tanto brasileiras quanto estrangeiras ${ }^{7-9}$.

Quanto às atitudes e opiniões, destaca-se a percepção dos acadêmicos sobre o papel que os profissionais de saúde desempenham como modelos de comportamento. A porcentagem de estudantes que tem essa opinião na USS foi de 78,07\%. Em outras instituições esse percentual variou entre $62,90 \%$ (Florianópolis - SC, 2007) e 69,93\% (Campo Grande - MS, 2006) ${ }^{7,8}$. Ainda que a porcentagem da USS seja superior à encontrada em outras instituições, fica evidente que um número significativo de estudantes ainda não está imbuído do seu real papel como modelo de comportamento para os pacientes e para o público em geral ${ }^{8}$. Essa percepção sofre influência direta do fato de o respondente ser ou não tabagista.

O principal objetivo deste trabalho foi verificar se a atitude dos estudantes de cursos da área da saúde frente ao paciente que fuma é ou não modificada pelo fato de o aluno ser ou não tabagista. As questões que melhor atingem este objetivo perguntaram se os profissionais da saúde devem rotineiramente aconselhar pacientes fumantes a pararem de fumar, se eles têm o papel de fornecer conselhos e informações sobre como parar de fumar, e se profissionais que fumam são menos propensos a aconselhar seus pacientes a pararem de fumar. Dentre estas três questões, apenas as respostas dadas à segunda não tiveram diferença significativa comparando-se estudantes fumantes e não fumantes. Isto demonstra o impacto que o tabagismo tem sobre a atitude dos estudantes de profissões de saúde, o que é alarmante, considerando-se o papel fundamental que esses futuros profissionais terão na luta contra o consumo de tabaco ${ }^{2}$.

Ainda que a maioria dos estudantes fumantes $(63,64 \%)$ acreditem que não, evidências demonstram que o médico que fuma tem uma menor tendência de abordar o uso do tabaco durante as consultas com seus pacientes ${ }^{10}$.

Com relação ao currículo do curso e treinamento recebido, apenas $24,56 \%$ dos estudantes afirmam ter recebido algum tipo de treinamento formal em 
técnicas de abordagem para abandono do fumo para serem usadas com os pacientes. É um percentual assustadoramente baixo, mas que não difere dos dados já publicados. No Brasil, o percentual se situa entre 21,54\% (Campo Grande - MS, 2006) e 34,74\% (Juiz de Fora - MG, 2007) ${ }^{7,8}$. No mundo todo, a porcentagem é de aproximadamente $24 \%$. Esses resultados sugerem que as instituições de ensino superior têm falhado ao não incluir em seus currículos o treinamento necessário a esses futuros profissionais sobre técnicas de cessação, mesmo com todas as evidências de que profissionais treinados são mais propensos a intervir nos casos de pacientes que consomem tabaco do que os não treinados ${ }^{2,9}$.

\section{Conclusões}

Considerando-se a alta prevalência de tabagismo encontrada entre acadêmicos da USS, a influência que esse hábito tem sobre a atitude dos estudantes diante de pacientes fumantes e também o papel vital que esses futuros profissionais de saúde têm a desempenhar na luta pela cessação do uso do tabaco, conclui-se que mais ênfase deve ser destinada ao treinamento que recebem durante seus cursos de graduação.

Além do treinamento formal, as instituições de ensino devem também fornecer auxílio e orientação a seus próprios alunos fumantes para que interrompam esse hábito. Esta medida se torna ainda mais importante quando se verifica o forte impacto que o tabagismo tem sobre a atuação dos profissionais de saúde para com pacientes fumantes.

\section{Declarações}

Os autores declaram não possuírem conflitos de interesse diretos ou indiretos.

Parte do presente trabalho foi apresentado na forma de pôster no XV Encontro de Iniciação Científica da Universidade Severino Sombra, em outubro de 2016.

\section{Comitê de Ética}

Este trabalho recebeu aprovação do Comitê de Ética em Pesquisa da Universidade Severino Sombra. Número do parecer: 1.144.114, de 13 de maio de 2015.

\section{Agradecimentos}

À FUSVE/USS pela bolsa de PIBIC. Aos Coordenadores e alunos dos cursos envolvidos na pesquisa pela colaboração.

\section{Notas}

a. Material apresentado no Encontro Estadual de Vigilância de Doenças Crônicas Não Transmissíveis, promovido pela Secretaria de Saúde do Estado do Rio de Janeiro e realizado em 9 de novembro de 2016, na sede da Secretaria, no Rio de Janeiro.

\section{Referências}

1.World Health Organization. Report on the global tobacco epidemic, 2013: enforcing bans on tobacco advertising, promotion and sponsorship. Genebra: World Health Organization, 2013.

2. Lancaster $\mathrm{T}$ et al. Effectiveness of interventions to help people stop smoking: findings from the Cochrane library. British Medical Journal, London, 2000; 321( 7257): 355-358.

3. Department of Health and Human Services. Public Health Service.Treating Tobacco Use and Dependence: 2008 Update. Rockville: U.S. Department of Health and Human Services, 2008.

4.Botelho $\mathrm{C}$ et al. Tabagismo em universitários de ciências da saúde: prevalência e conhecimento. Jornal Brasileiro de Pneumologia, 2011; v. 37(3):360-366.

5.Warren CW et al. Tobacco use and cessation couseling among pharmacy students. Journal of Behavioral Health, Ankara, 2013; 2(1): 8-18.

6.Paceli R et al. Prospective analysis among medical school of University of São Paulo: GHPSS (2008/2011). European Respiratory Journal, Sheffield, 2012; 40(56): 4062.

7. Center For Disease Control And Prevention. Global Tobacco Surveillance System Data (GTSSData).FactSheets.

8.Ministério da Saúde. Instituto Nacional do Câncer. A situação do tabagismo no Brasil: Dados dos inquéritos do Sistema Internacional de Vigilância do Tabagismo da Organização Mundial da Saúde realizados no Brasil entre 2002 e 2009.Rio de Janeiro: Instituto Nacional do Câncer, 2011.

9. Warren CW et al. The GTSS Atlas. Brighton: CDC Foundation, 2009.

10. Pipe A, Sorensen M, Reide R. Physician smoking status, attitudes toward smoking, and cessation advice to patients: An international survey. Patient Education and Couseling, Philadelphia, 2009;74(1):118-123. 